\title{
Comprehensive transcriptome analysis of peripheral blood unravels key IncRNAs implicated in ABPA and asthma
}

\author{
Chen Huang ${ }^{1,2}$, Dongliang Leng ${ }^{3}$, Peiyan Zheng ${ }^{4}$, Min Deng ${ }^{3}, \mathrm{Lu} \mathrm{Li}^{4}$, Ge Wu ${ }^{4}$, Baoqing Sun ${ }^{4}$, Xiaohua Douglas \\ Zhang ${ }^{\text {Corresp. } 3}$ \\ ${ }^{1}$ Dr. Neher's Biophysics Laboratory for Innovative Drug Discovery, Macau University of Science and Technology, Macau, SAR, China, Macau, China \\ Stat Key laboratory of Quality Research in Chinese Medicine, Macau Institute For Applied Research in Medicine and Health, Macau University of Science \\ and Technology, Macau, SAR, China, Macau, China \\ 3 Faculty of Health and Science, University of Macau, Macao, Macau \\ 4 Department of Allergy and Clinical Immunology, State Key Laboratory of Respiratory Disease, National Clinical Research Center of Respiratory Disease, \\ Guangzhou Institute of Respiratory, Health, First Affiliated Hospital of Guangzhou Medical University, Guangzhou, Guangdong, China \\ Corresponding Author: Xiaohua Douglas Zhang \\ Email address: douglaszhang@um.edu.mo
}

Allergic bronchopulmonary aspergillosis (ABPA) is a complex hypersensitivity lung disease caused by a fungus known as Aspergillus fumigatus. It complicates and aggravates asthma. Despite their potential associations, the underlying mechanisms of asthma developing into ABPA remain obscure. Here we performed an integrative transcriptome analysis based on three types of human peripheral blood, which derived from ABPA patients, asthmatic patients and health controls, aiming to identify crucial IncRNAs implicated in ABPA and asthma. Initially, a high-confidence dataset of IncRNAs was identified using a stringent filtering pipeline. A comparative mutational analysis revealed no significant difference among these samples. Differential expression analysis disclosed several immune-related mRNAs and IncRNAs differentially expressed in ABPA and asthma. For each disease, three sub-networks were established using differential network analysis. Many key IncRNAs implicated in ABPA and asthma were identified, respectively, i.e., AL139423.1-201, AC106028.4-201, HNRNPUL1-210, PUF60-218 and SREBF1-208. Our analysis indicated that these IncRNAs exhibits in the loss-of-function networks, and the expression of which were repressed in the occurrences of both diseases, implying their important roles in the immune-related processes in response to the occurrence of both diseases. Above all, our analysis proposed a new point of view to explore the relationship between ABPA and asthma, which might provide new clues to unveil the pathogenic mechanisms for both diseases. 


\section{Comprehensive transcriptome analysis of peripheral blood unravels key}

\section{Chen Huang ${ }^{1,2 \dagger}$, Dongliang Leng ${ }^{3 \dagger}$, Peiyan Zheng ${ }^{4}$, Min Deng ${ }^{3}$, Lu Li ${ }^{4}, \mathrm{Ge} \mathrm{Wu}^{4}$, Baoqing} Sun $^{4 *}$, xiaohua Douglas Zhang ${ }^{3 *}$

${ }^{1}$ Dr. Neher's Biophysics Laboratory for Innovative Drug Discovery, Macau University of Science and Technology, Macau, SAR, China

${ }^{2}$ Stat Key laboratory of Quality Research in Chinese Medicine, Macau Institute For Applied Research in Medicine and Health, Macau University of Science and Technology, Macau, SAR,

${ }^{4}$ Department of Allergy and Clinical Immunology, State Key Laboratory of Respiratory Disease, National Clinical Research Center of Respiratory Disease, Guangzhou Institute of Respiratory Health, First Affiliated Hospital of Guangzhou Medical University, Guangzhou, Guangdong, China 


\section{ABSTRACT}

26 Allergic bronchopulmonary aspergillosis (ABPA) is a complex hypersensitivity lung disease

27 caused by a fungus known as Aspergillus fumigatus. It complicates and aggravates asthma. Despite

28 their potential associations, the underlying mechanisms of asthma developing into ABPA remains

29 obscure. Here we performed an integrative transcriptome analysis based on three types of human

30 peripheral blood, which derived from ABPA patients, asthmatic patients and health controls,

31 aiming to identify crucial lncRNAs implicated ABPA and asthma. Initially, a high-confidence

32 dataset of lncRNAs was identified using a stringent filtering pipeline. A comparative mutational

33 analysis revealed no significant difference among these samples. Differential expression analysis

34 disclosed several immune-related mRNAs and lncRNAs differentially expressed in ABPA and

35 asthma. For each disease, three sub-networks were established using differential network analysis.

36 Many key lncRNAs implicated in ABPA and asthma were identified, respectively, i.e.,

37 AL139423.1-201, AC106028.4-201, HNRNPUL1-210, PUF60-218 and SREBF1-208. Our

38 analysis indicated that these lncRNAs exhibits in the loss-of-function networks, and the expression

39 of which were repressed in the occurrences of both diseases, implying their important roles in the

40 immune-related processes in response to the occurrence of both diseases. Above all, our analysis

41 proposed a new point of view to explore the relationship between ABPA and asthma, which might

42 provide new clues to unveil the pathogenic mechanisms for both diseases. 
43 Trial registration: gyfyy-2016-61. Registered 27 October 2016.

44 Keyword: Long noncoding RNAs, ABPA, asthma, RNA sequencing, transcriptome analysis, network analysis

\section{INTRODUCTION}

Allergic bronchopulmonary aspergillosis (ABPA) is a Th2 hypersensitivity disease response to the presence of Aspergillus fumigatus in the bronchial mucosa. The first case was described by Hinson et al. in 1952 in the United Kingdom (Hinson et al. 1952). The prevalence of ABPA was initially

51 thought to be rare, which was estimated to be occurred in 1 2\% in asthmatic patients (Singh et al. 2018). However, increasing evidences indicate that ABPA is underdiagnosed and much more prevalent than previously estimated (Greenberger 2002; Patel et al. 2019). Denning et al estimates that global prevalence of ABPA may be $0.7-3.5 \%$ of asthmatic patients (Denning et al. 2013), but now be increased into $2.5 \%$ (Patel et al. 2019). On the other hand, asthma is a common lung condition characterized by bronchial hyper-responsiveness and variable airflow obstruction. A few cases exhibit complicated and uncontrolled symptoms. This type of asthma is defined as severe asthma, which could be closely linked to atopic to airborne allergens, especially to fungal spores 
60 fungi causing fungal sensitization (Agarwal 2011; Agarwal \& Gupta 2011), suggesting close

61 association between ABPA and asthma.

62 In spite of the fact that the correlation between asthma and ABPA has been well elucidated, the

63 detailed causality between two entities, particularly the pathogenesis of ABPA is not fully

64 understood yet. For instance, only a small proportion of severe asthmatic patients develop ABPA.

65 Both diseases are characterized by extremely high serum level of IgE (Immunoglobulin E) but a

66 small proportion of ABPA patients has less high serum IgE level (Agarwal et al. 2019).

67 Approximately $60 \%$ of ABPA patients can benefit from traditional treatment for allergic fungal

68 disorder, e.g., the use of systemic oral itraconazole, but a few patients exhibit uncontrolled

69 symptoms despite various treatments have been tried.

70 Recently, some progress has been made in disclosing genetic polymorphisms associated with

71 ABPA risk and progression. A typical example indicates that asthmatic patients expressing HLA-

72 DR2 and/or DR5 and lacking HLA-DQ2 are susceptible to develop ABPA after exposure to $A$.

73 fumigatus (Chauhan et al. 2000). Around half of non-ABPA atopic Aspergillus-sensitive

74 individuals are found to possess HLA-DR2 and/or HLADR5 genotype, whereas the HLA-

75 DRB1*1501 and HLA-DRB1*1503 genotype are reported to have high relative risk (Knutsen

76 2017). The mutations in cystic fibrosis transmembrane conductance regulator gene (CFTR) is

77 reported to raise the risk of ABPA in asthmatic patients (Agarwal et al. 2012). 
78 Despites there are some advances in the disclosure of genetic risk underlying ABPA, there are still

79 gaps to be filled. Typically, ABPA patients are found to harbor high frequency of polymorphism

80 in Toll-like receptor 9T-1237C (TLR9T-1237C). However, the polymorphisms of TLR9 are

81 reported to have no associations with the patients with severe asthma who are also associated with

82 Aspergillus sensitivity (Carvalho et al. 2008). It was suggested that genetic variations are not the

83 only factor contributing ABPA pathogenesis. Increasing evidences demonstrated that

84 transcriptome dysfunction and aberrant gene expression also have a critical role in disease

85 pathogenesis (Zeller et al. 2010). Particularly, lncRNAs are proved to be key regulatory layers

86 associated with asthma. For instance, lncRNAs BCYRN1 is found to promote the proliferation and

87 migration of rat airway smooth muscle cells in asthma via activation of transient receptor potential

881 (Zhang et al. 2016). Here, aimed at identifying potential lncRNAs implicated in the occurrence

89 of ABPA and asthma, as well as their possible roles in both diseases, we performed a

90 comprehensive transcriptome analysis on the three different types of human peripheral blood,

91 including ABPA, asthma and health controls. Our analysis discovered many key lncRNAs which

92 are likely to be related to immune functions and might play key roles in the pathogenesis of ABPA

93 and asthma. Our findings benefit the discovery of novel biomarkers and targets guiding for

94 diagnosis and therapy for both diseases. 


\section{MATERIALS AND METHODS}

\section{Inclusion, Sampling and deep RNA sequencing}

98 This study was approved by the Medical Ethics Committee of First Affiliated Hospital of

99 Guangzhou Medical University (ethics approval no. gyfyy-2016-73). All experiments were

100 performed in accordance with relevant guidelines and regulations of the Ethics Committee of First

101 Affiliated Hospital of Guangzhou Medical University. All participants provided written informed

102 consent prior to the publication of clinical and sequencing data. Briefly, a total of 27 unique male

103 individuals were enrolled for our study, including 7 asthma patients (diagnosed as allergic asthma),

10412 ABPA patients and 8 healthy individuals considered as control group (Table 1). The diagnosis

105 of asthma was according to the latest Global Initiative for Asthma (GINA) guidelines, and the

106 diagnosis of ABPA was based on the criteria of The International Society of Human and Animal

107 Mycology (ISHAM) working group, which contains 2 obligatory criteria and 3 additional criteria

108 (Shah \& Panjabi 2016). Based on the clinical symptoms, 5 asthma patients were at exacerbation

109 stage and 2 were at chronic stage (Table S1). And according to the proposed clinical staging of

110 ABPA (Shah \& Panjabi 2016), among the included ABPA patients, 7 were at acute stage, 1 was

111 at exacerbation stage, 2 was at response stage, while the other 2 were not able to assess the clinical

112 stage because of insufficient clinical data (Table S1). 
113 For each enrolled subject, the peripheral whole blood was extracted and the peripheral blood

114 mononuclear cell (PBMC) was separated immediately by ficoll-paque. The total RNA was

115 extracted using trizol (invitrogen) method and the library for RNA sequencing was prepared based

116 on a standard protocol established by RiboBio Company in Guangzhou and sequenced on Illumina.

117 All the raw RNA-seq data used in the present study are deposited at Short Read Archive (SRA)

118 database of NCBI (https:/trace.ncbi.nlm.nih.gov/Traces/sra/) and are assigned the accession

119 number PRJNA582337.

\section{Raw data processing}

121 The raw data was trimmed by Trimmomatic v0.36 (ILLUMINACLIP: TruSeq3-

122 PE.fa:2:30:10:8:true SLIDINGWINDOW:4:15 LEADING:3 TRAILING:3 MINLEN:50) (Bolger

123 et al. 2014) and the cleaned reads were aligned against Ensembl hg38 human genome via STAR

124 (v020201) (Dobin et al. 2013). The transcriptome was re-constructed using StringTie (v1.3.3b)

125 (Pertea et al. 2015). A stringent stepwise pipeline (Figure 1) which had been applied in our

126 previous studies (Huang et al. 2019a; Leng et al. 2019; Zheng et al. 2020) was utilized for

127 identification of high-confidence dataset of lncRNAs. 


\section{Identification of IncRNAs}

129 Firstly, the known lncRNAs were picked out from complete set of assembled transcripts according

130 to the "biotype_transcript" of reference gtf file of Homo sapiens from Ensembl database (Zerbino

131 et al. 2018). The transcript was detected as lncRNA when its "biotype transcript" tagged as

132 "Long_non-coding_RNA", "Non_coding", “3prime_overlapping_ncRNA", “Antisense",

133 "lincRNA", "Retained_intron", "Sense_intronic", "Sense_overlapping", "macro_lncRNA" and

134 "bidirectional_promoter_lncRNA".

135 Then the human known mRNAs derived from Ensembl database were excluded from the

136 remaining transcripts. Afterwards, the alignment was performed for the remaining transcripts

137 against known protein sequences from NCBI nr database (Pruitt et al. 2007) and Uniprot database

138 (UniProt Consortium 2018). Successfully aligned transcripts were excluded from potentially non-

139 protein-coding sequences. For the unmapped transcripts, filtering was conducted to get rid of the

140 sequences with length less than $200 \mathrm{nt}$ and longest ORF longer than 100 residues. Finally, the

141 qualified sequences were further filtered to remove the protein-coding sequences according to the

142 results from Pfamscan (El-Gebali et al. 2019) and CPC (Kong et al. 2007). Eventually, the

143 remaining transcripts were reserved as final dataset of lncRNAs. 


\section{Transcriptome variations analysis}

145 For single nucleotide variants, i.e., SNPs and small insertion and deletion (INDEL) detection, we

146 applied Genome Analysis Toolkit (GATK) analysis pipeline (Van der Auwera et al. 2013) for

147 variant calling. All the variants were annotated by ANNOVAR (Wang et al. 2010). Simple

148 sequence repeats (SSRs) detection was performed using the Perl script of MISA-MicroSAtellite

149 identification tool (http://pgrc.ipk-gatersleben.de/misa/) with default parameters. Statistical

150 hypotheses were used to investigate whether there were significant differences in the observed

151 proportion of SNPs, INDELs and SSRs among populations of ABPA, asthma and healthy control.

152 The detailed process sees Supplementary methods.

\section{Transcriptome expression analysis}

154 Differential expression analysis was performed using $\mathrm{R}$ package DESeq2 (adjusted p-value

$155 \leqslant 0.05)($ Love et al. 2014). Functional enrichment analysis was performed via ClueGO V2.5.2

156 (Bindea et al. 2009) plugin in Cytoscape V3.6.1 (Shannon et al. 2003), which could generate a

157 dynamical network structure composed of functionally grouped terms based on the gene list of

158 interest, i.e., differentially expressed genes. The differentially expressed transcripts were subjected

159 to R programming language for hierarchical cluster analysis to detect distinct expression patterns. 
160 Differential interaction network analysis was conducted based on three filtering criteria, which

161 generated three sub-networks for each group, including loss-of-function network, gain-of-function

162 network, and anti-function network. 12 algorithms (MCC, DMNC, MNC, Degree, EPC,

163 BottleNeck, EcCentricity, Closeness, Radiality, Betweenness, Stress, ClusteringCoefficient) of

164 cytoHubba (Chin et al. 2014) plugin in Cytoscape were used to identify hub node for the

165 established network. Detailed procedure see Supplementary Methods. For the hub node validation,

166 the expression profile of four data sets (GSE35571, GSE473, GSE31773, GSE2125) from NCBI

167 GEO database were downloaded and analyzed (Table S2).

168 Statistics of clinical data

169 For the basic statistics of clinical data (supplementary file 3), all the data were shown in median

170 (interquartile range). Non-parametric Kruskal-Wallis test was performed to compare the

171 differences between three groups while Mann-Whitney test was performed to compare the

172 differences between two groups. Regarding the ratio data, Chi-square test was applied for the

173 comparison. Statistical analysis was performed by SPSS 22.0. A value of $P<0.05$ was considered

174 as statistically significant. 


\section{RESULTS}

\section{Characteristics of study subjects}

177 As shown in Table 1, the asthma group were elder compared with either ABPA group or Healthy

178 control group $(\mathrm{P}<0.05)$. There was no significant difference in eosinophil or neutrophil counts of

179 peripheral blood in the three groups. Regarding the percentages of inflammatory cells in induced

180 sputum, no statistical difference was found in any type of inflammatory cells between asthma and

181 ABPA. There was also no statistical difference in the results of lung function between the two

182 patient groups. In the comparison of IgE levels, patients with ABPA had higher total IgE levels (P

$183<0.05)$, higher positive rate of specific $\operatorname{IgE}(\mathrm{P}<0.05)$, in particular A. fumigatus specific $\operatorname{IgE}(\mathrm{P}<$

184 0.01) than patients with asthma. In addition, use of glucocorticoids (either oral or inhaled) did not

185 differ significantly between asthma and ABPA.

\section{LncRNAs identification}

187 RNA-seq via Illumina sequencing platform yielded on average 130 million reads per sample

188 (Table S3). After quality trimming, the clean reads were mapped to human genome and

189 subsequently assembled into 283,883 transcripts. A total of $94,155 \operatorname{lncRNAs}$ across 27 samples,

190 including 52,791 known lncRNAs and 41,364 novel lncRNAs were detected using a stringent

191 stepwise filter pipeline (Figure 1). 
192 No significant difference in variants across ABPA, asthma and health controls

193 A total of 4,521,799 SNPs and 671,669 INDELs were identified across 27 samples via GATK

194 analysis pipeline (Figure 1, Table S4). Majority of variants locates in intronic ( 61\%) and

195 intergenic regions ( $26.2 \%$ ) (Table 2, Figure S1). 62,733 variants are in genic regions, and $42.9 \%$

196 of them are synonymous SNP (Table S5). 44,090 variants significantly existed differentially across

197 groups by Fisher Exact probability test (Supplementary file 1). In addition, many genes (including

198 few lncRNAs) exhibited distinct variants cross groups (Figure 2A).

199 For SSRs analysis, a total of 4,740,915 different types of SSRs were identified $(175,589$ per sample

200 on average) (Table S6). No significant difference in SSRs across these samples was detected

201 (Figure 2D-I), despites two statistical approaches (details process see Supplementary Methods)

202 revealed around 150 SSRs were differentially enriched in total (Figure 2B-C, Supplementary file 203 2).

204 Differential expression analysis reveals immune-related processes implicated in both 205 diseases

2062,481 differentially expressed transcripts (DETs, including 554 lncRNAs) were found in the

207 comparison of ABPA vs healthy controls (Figure 3A, D), and 2,706 transcripts (including 529

208 lncRNAs) were differentially expressed in the comparison of asthma vs healthy controls,

209 respectively. Functional analysis via ClueGO for both DET datasets demonstrated that many 
210 immune-related biological processes are significantly enriched, including leukocyte activation,

211 activation of innate immune response and positive regulation of immune effector process, etc

212 (Figure 3B, C). Furthermore by the comparison of two DET datasets, distinct DETs involved in

213 diverse biological processes were detected among three different groups (Figure 3D, E).

214 Additionally, hierarchical cluster analysis via R language based on DETs revealed three main

215 expression patterns of DETs cross these samples (Figure 4). Concretely, many transcripts

216 (including mRNAs and lncRNAs) are found to be down-regulated in healthy controls but were up-

217 regulated in ABPA and asthma patients (Figure 4A). Functional analysis showed that they might

218 be involved in myeloid leukocyte mediated immunity, regulation of defense response to virus, etc.

219 (Figure 4B). On the contrary, several transcripts are down-regulated in healthy controls but are up-

220 regulated in ABPA and asthma patients (Figure 4E), which might be associated with

221 columnar/cuboidal epithelial cell differentiation, regulation of apoptotic signaling pathway, etc.

222 (Figure 4F). A few transcripts which are specifically up-regulated in ABPA patients (Figure 4C),

223 and their functions might be correlated to supramolecular fiber organization and positive

224 regulation of organelle organization (Figure 4D).

225 Differential network analysis reveals crucial IncRNAs correlated to both diseases

226 The aforementioned analysis has revealed several lncRNAs and mRNAs differentially expressed

227 in ABPA and asthma patients. Next to investigate the possible roles of these lncRNAs implicated 
228 in the pathogenesis of ABPA and asthma were still unclear, we proposed a differential network

229 analysis. Initially, we built an RNA-RNA interaction network based on the expression profile data

230 of 8 healthy controls using Pearson Correlation Coefficient (PCC) method. Then the interactions

231 of these RNAs in the networks were re-assessed based on the expression profiles of 12 ABPA

232 patients and 7 asthmatic patients. To assess the changes of network from healthy status to disease

233 status, three different filtering criteria (details see Supplementary Methods) was proposed to

234 construct three sub-networks for each disease, namely loss-of-function network, gain-of-function

235 network, and anti-function network.

236 In loss-of-function network, for instance, 293,926 RNA-RNA interactions with strong correlation

237 ( $\mid$ PCC value $\mid \geq 0.70 \&$ P-value $<0.05)$ are found in health controls, whereas these interactions are

238 shown having weak correlation $(\mid \mathrm{PCC}$ value $\mid \leq 0.30)$ in ABPA patients. It is suggested these RNA-

239 RNA interactions might be disrupted by the occurrence of ABPA. 243,399 RNA-RNA interactions

240 exhibit similar situation in asthma. For each disease, the immune-related RNA-RNA interactions

241 in two loss-of-function networks were extracted to establish the loss-of-immune-related-function

242 network, respectively (Figure 5-A, B). Key elements (hub nodes of the network) in both networks

243 were detected by topological network analysis via Cytohubba. Our analysis revealed several hub

244 lncRNAs which might play key roles in the immune dysfunction of ABPA and asthma. In this 
245 way, we built the gain-of-function and anti-function networks for both diseases (Figure 5 C, D, E,

246 F), and relevant key lncRNAs and mRNAs were disclosed (Table S7, S8).

247 To further investigate convincing RNA-RNA interactions implicated in ABPA and asthma, we

248 integrated the above-mention networks (Figure S2). Notably, the RNAs in the network can be

249 categorized into four main immune-related groups, including leukocyte involved immune process,

250 cytokine related immune process, autophagy apoptosis regulation and response to exogenous

251 invasion. Topological analysis identified many key mRNAs/lncRNAs implicated in the regulation

252 of diverse immune-related processes in response to ABPA and asthma (the frequency of counting

253 as top 20 hub nodes among 12 algorithms $\geq 3$, Table 3, details see Supplementary Methods),

254 including five lncRNAs, namely AL139423.1-201, AC106028.4-201, HNRNPUL1-210, PUF60-

255218 and SREBF1-208, and five known mRNAs (PRR14L-201, CCRL2-202, GDAP2-202,

256 SPAG1-206, SLC35B2-207). Notably, many mRNAs have been already demonstrated to be

257 associated to asthma or ABPA by previous studies. For instance, Inhibition of PARP14 (Top one

258 hub node in Table 3, it was found to significantly up-regulated in both diseases) was reported to

259 reduce allergic airway diseases, it thereby was proposed as potential therapeutic target for asthma

260 (Mehrotra et al. 2013).

261 Moreover, all the RNA-RNA interactions involved the five key lncRNAs were observed in the

262 loss-of-function networks in both diseases and exhibited down-regulated expression in the 
263 peripheral blood of patients in both diseases (Table 3), suggesting the regulations of hub lncRNAs

264 in these interacted target RNAs were disrupted after the occurrence of asthma or ABPA. Their

265 targets were found to be involved in many immune-related processes and pathways (Figure 6A-

266 E). Permutation test shows majority of hub nodes are high-confidence (Details see Supplementary

267 Methods). These findings indicate that the expression of these five lncRNAs would be repressed

268 during the occurrences of ABPA or asthma, many immune-related processes mediated by them

269 might be disrupted, simultaneously.

\section{Validation of hub nodes using GEO datasets}

271 Four hub nodes including two mRNAs (ENST00000327423, ENST00000369443) and two

272 IncRNAs (ENST00000587128, ENST00000595806) from the two networks were selected for

273 validation. The expression profiles of four GEO datasets (GSE35571, GSE473, GSE31773 and

274 GSE2125) were used. The results indicated that the expression level of the selected hub nodes are

275 consistent with the result of GEO datasets (Figure 6F-I). Also, many interactions involved in these

276 hub nodes could be found in these GEO datasets (Table S9), implying our predictions are reliable

277 and convincing. 


\section{DISCUSSIONS}

279 ABPA has not received the importance that it deserves despites it occurs with a world-wide

280 distribution in a great number of patients with asthma or cystic fibrosis (CF). Although more than

281 half of century has passed since ABPA was first described, its exact pathogenesis as well as

282 potential relationship with asthma is still unknown. The clinical data of the collected subjects in

283 the present study do not show significant difference between ABPA and asthmatic patients, except

284 that ABPA have higher IgE level (Table 1). We thereby try to find some clues from the

285 transcriptome. Initially, we looked into whether there is mutational difference between ABPA,

286 asthma and health controls. The results showed that many genes (including two lncRNAs:

287 LINC00398, LINC00892) exhibited distinct mutations cross those samples (Figure 2A).

288 Regarding SSRs, no difference in biological significance was found cross those samples, despites

289 some of them showed significant $\mathrm{P}$ value in statistic test after filtering. These findings indeed are

290 consistent to the mainstream view on the pathophysiological mechanisms of ABPA and asthma,

291 i.e., unlike genetic diseases that mutations largely contribute to the pathogenesis of these diseases,

292 the development of ABPA or asthma should involve the complex cross-talking between

293 specific heredities and environmental factors. Mutations particularly in transcriptome might have

294 only a marginal effect on the occurrence of both diseases. This finding actually is consistent with

295 other previous studies, which indicated that genetic risk factors have overall small effects for the 
296 adult-onset asthma, implying a greater role for non-genetic risk in adult-onset asthma (Pividori et

297 al. 2019).

298 Next, we try to find some clues from the comparison of gene expression among those groups,

299 particularly in lncRNAs. In addition to traditional differential expression analysis, we proposed a

300 differential network analysis to detect the dynamic changes of transcriptome across those different

301 groups. Differential network analysis based on three different filtering criteria figured out several

302 key lncRNAs and mRNAs. The reason that we applied different filtering criteria for three different

303 groups is that three networks exhibit extremely distinct threshold value of pearson correlation

304 coefficient, and different criteria would yield a similar number of RNA-RNA interactions in

305 different groups. Notably, we applied 12 different algorithms of topological network analysis to

306 make sure our predictions more reliable and convincing. Our analysis indicated that the

307 interactions involved in 82 key transcripts (hub nodes in network) were dramatically disrupted by

308 the occurrence of ABPA, and some interactions were repressed by ABPA and some were gained

309 in response to ABPA (Table S7). For instance, the gene RGS3 (Top $2^{\text {th }}$ hub node in Table S7)

310 encodes the regulator of G-protein signaling 3, and it can activate MAP kinases (MAPK), which

311 was found to be critically involved in modulation of asthmatic inflammation. It also was proposed

312 for a potential therapeutic target for asthma (Huang et al. 2019b). The gene GPR171 (Top $3^{\text {th }}$ hub

313 node in Table S7) encodes probable G-protein coupled receptor, which was reported to be 
314 implicated in asthma endophenotypes and negatively regulate myeloid cell differentiation

315 (Thompson et al. 2006).Similarly, we identified 93 key mRNAs/lncRNAs (Table S8) in asthma

316 network. Few hub nodes share in ABPA and asthma networks, suggesting that these distinct hub

317 nodes might be good candidates for further investigating the difference between ABPA and

318 asthma. Some mRNAs/lncRNAs were found as new risk factors associated with ABPA or asthma,

319 i.e., AL139423.1-201, GDAP2, which might be novel biomarkers and targets for diagnosis and

320 therapy in both diseases.

321 By integrating two networks, we could find several common key mRNAs and lncRNAs.

322 Permutation test shows the reliability of this analysis. Some of them have already been

323 demonstrated to be correlated to asthma or ABPA. For example, PARP14 was found to play a role

324 in transcription of interleukin-4 (IL4)-responsive genes, which controls cell survival, metabolism

325 and proliferation (Cho et al. 2009). Inhibition of PARP14 was found to reduce allergic airway

326 diseases (38). CCRL2 (Top $4^{\text {th }}$ hub node in Table 3) encodes a chemokine receptor like protein.

327 Chemokines and their receptors have reported to mediate signal transduction, which are critical

328 for the recruitment of effector immune cells to the site of inflammation (D'Ambrosio et al. 2001).

329 More importantly, many known and novel lncRNAs were identified to be related to asthma and

330 ABPA for the first time, i.e., AL139423.1-201, HNRNPUL1-210, AC106028.4-201, etc. 


\section{CONCLUSIONS}

333 Our analysis discloses many lncRNAs associated to immune-related mRNAs, which suggests

334 these lncRNAs might participate regulation of immune-related processes in response to the

335 occurrence of both diseases. Further investigation on the interactions among them might provide

336 some clues to access underlying mechanisms of pathogenesis for both diseases. Certainly, more

337 ingenious experimental design and validation is required to conclude the concrete roles of these

338 lncRNAs in both diseases. In short, our analysis describes a rough transcriptome landscape of

339 ABPA and asthma, and benefits understanding the pathogenesis of both diseases.

\section{REFERENCES}

341 Agarwal R. 2011. Severe asthma with fungal sensitization. Curr Allergy Asthma Rep 11:403-413. 10.1007/s11882-011-0217-4

Agarwal R, and Gupta D. 2011. Severe asthma and fungi: current evidence. Med Mycol 49 Suppl 1:S150157. $10.3109 / 13693786.2010 .504752$

Agarwal R, Khan A, Aggarwal AN, and Gupta D. 2012. Link between CFTR mutations and ABPA: a systematic review and meta-analysis. Mycoses 55:357-365. 10.1111/j.1439-0507.2011.02130.x

Agarwal R, Sehgal IS, Dhooria S, and Aggarwal AN. 2019. Challenging cases in fungal asthma. Med Mycol 57:S110-S117. 10.1093/mmy/myy063

Bindea G, Mlecnik B, Hackl H, Charoentong P, Tosolini M, Kirilovsky A, Fridman WH, Pages F, Trajanoski Z, and Galon J. 2009. ClueGO: a Cytoscape plug-in to decipher functionally grouped gene ontology and pathway annotation networks. Bioinformatics 25:1091-1093. 10.1093/bioinformatics/btp101

Bolger AM, Lohse M, and Usadel B. 2014. Trimmomatic: a flexible trimmer for Illumina sequence data. Bioinformatics 30:2114-2120. 10.1093/bioinformatics/btu170

Carvalho A, Pasqualotto AC, Pitzurra L, Romani L, Denning DW, and Rodrigues F. 2008. Polymorphisms in toll-like receptor genes and susceptibility to pulmonary aspergillosis. J Infect Dis 197:618-621. 10.1086/526500 
358 Chauhan B, Santiago L, Hutcheson PS, Schwartz HJ, Spitznagel E, Castro M, Slavin RG, and Bellone CJ.

359

360

361

362

363

364

365

366

367

368

369

370

371

372

373

374

375

376

377

378

379

380

381

382

383

384

385

386

387

388

389

390

391

392 2000. Evidence for the involvement of two different MHC class II regions in susceptibility or protection in allergic bronchopulmonary aspergillosis. J Allergy Clin Immunol 106:723-729. 10.1067/mai.2000.109913

Chin $\mathrm{CH}$, Chen SH, Wu HH, Ho CW, Ko MT, and Lin CY. 2014. cytoHubba: identifying hub objects and sub-networks from complex interactome. BMC Syst Biol 8 Suppl 4:S11. 10.1186/1752-0509-8-S4S11

Cho SH, Goenka S, Henttinen T, Gudapati P, Reinikainen A, Eischen CM, Lahesmaa R, and Boothby M. 2009. PARP-14, a member of the B aggressive lymphoma family, transduces survival signals in primary B cells. Blood 113:2416-2425. 10.1182/blood-2008-03-144121

D'Ambrosio D, Mariani M, Panina-Bordignon P, and Sinigaglia F. 2001. Chemokines and their receptors guiding T lymphocyte recruitment in lung inflammation. Am J Respir Crit Care Med 164:12661275. 10.1164/ajrccm.164.7.2103011

Denning DW, Pleuvry A, and Cole DC. 2013. Global burden of allergic bronchopulmonary aspergillosis with asthma and its complication chronic pulmonary aspergillosis in adults. Med Mycol 51:361370. 10.3109/13693786.2012.738312

Dobin A, Davis CA, Schlesinger F, Drenkow J, Zaleski C, Jha S, Batut P, Chaisson M, and Gingeras TR. 2013. STAR: ultrafast universal RNA-seq aligner. Bioinformatics 29:15-21. 10.1093/bioinformatics/bts635

El-Gebali S, Mistry J, Bateman A, Eddy SR, Luciani A, Potter SC, Qureshi M, Richardson LJ, Salazar GA, Smart A, Sonnhammer ELL, Hirsh L, Paladin L, Piovesan D, Tosatto SCE, and Finn RD. 2019. The Pfam protein families database in 2019. Nucleic Acids Res 47:D427-D432. 10.1093/nar/gky995

Greenberger PA. 2002. Allergic bronchopulmonary aspergillosis. J Allergy Clin Immunol 110:685-692. 10.1067/mai.2002.130179

Hinson KF, Moon AJ, and Plummer NS. 1952. Broncho-pulmonary aspergillosis; a review and a report of eight new cases. Thorax 7:317-333. 10.1136/thx.7.4.317

Huang C, Leng D, Lei KC, Sun S, and Zhang XD. 2019a. Transcriptome analysis reveals lncRNA-mediated complex regulatory network response to DNA damage in the liver tissue of Rattus norvegicus. $J$ Cell Physiol 234:23216-23231. 10.1002/jcp.28889

Huang LN, Sun L, Liu LM, Zhang HH, Liang ZB, Rui Y, Hu JF, Zhang Y, Christman JW, and Qian F. 2019b. p38alpha MAP kinase promotes asthmatic inflammation through modulation of alternatively activated macrophages. J Mol Cell Biol 11:1095-1097. 10.1093/jmcb/mjz054

Knutsen AP. 2017. Allergic bronchopulmonary aspergillosis in asthma. Expert Rev Clin Immunol 13:1114. $10.1080 / 1744666 X .2017 .1232620$

PeerJ reviewing PDF | (2020:12:56161:2:0:NEW 19 Apr 2021) 
393 Kong L, Zhang Y, Ye ZQ, Liu XQ, Zhao SQ, Wei L, and Gao G. 2007. CPC: assess the protein-coding

394

395

396

397

398

399

400

401

402

403

404

405

406

407

408

409

410

411

412

413

414

415

416

417

418

419

420

421

422

423

424

425

426

427

potential of transcripts using sequence features and support vector machine. Nucleic Acids Res 35:W345-349. 10.1093/nar/gkm391

Leng D, Huang C, Lei KC, Sun S, Sun B, and Zhang XD. 2019. Co-expression network analysis of lncRNAs and mRNAs in rat liver tissue reveals the complex interactions in response to pathogenic cytotoxicity. Int J Biol Sci 15:2296-2307. 10.7150/ijbs.33735

Love MI, Huber W, and Anders S. 2014. Moderated estimation of fold change and dispersion for RNA-seq data with DESeq2. Genome Biol 15:550. 10.1186/s13059-014-0550-8

Mehrotra P, Hollenbeck A, Riley JP, Li F, Patel RJ, Akhtar N, and Goenka S. 2013. Poly (ADP-ribose) polymerase 14 and its enzyme activity regulates $\mathrm{T}(\mathrm{H}) 2$ differentiation and allergic airway disease. J Allergy Clin Immunol 131:521-531 e521-512. 10.1016/j.jaci.2012.06.015

Patel AR, Patel AR, Singh S, Singh S, and Khawaja I. 2019. Treating Allergic Bronchopulmonary Aspergillosis: A Review. Cureus 11:e4538. 10.7759/cureus.4538

Pertea M, Pertea GM, Antonescu CM, Chang TC, Mendell JT, and Salzberg SL. 2015. StringTie enables improved reconstruction of a transcriptome from RNA-seq reads. Nat Biotechnol 33:290-295. $10.1038 /$ nbt. 3122

Pividori M, Schoettler N, Nicolae DL, Ober C, and Im HK. 2019. Shared and distinct genetic risk factors for childhood-onset and adult-onset asthma: genome-wide and transcriptome-wide studies. Lancet Respir Med 7:509-522. 10.1016/S2213-2600(19)30055-4

Pruitt KD, Tatusova T, and Maglott DR. 2007. NCBI reference sequences (RefSeq): a curated nonredundant sequence database of genomes, transcripts and proteins. Nucleic Acids Res 35:D61-65. 10.1093/nar/gk1842

Shah A, and Panjabi C. 2016. Allergic Bronchopulmonary Aspergillosis: A Perplexing Clinical Entity. Allergy Asthma Immunol Res 8:282-297. 10.4168/aair.2016.8.4.282

Shannon P, Markiel A, Ozier O, Baliga NS, Wang JT, Ramage D, Amin N, Schwikowski B, and Ideker T. 2003. Cytoscape: a software environment for integrated models of biomolecular interaction networks. Genome Res 13:2498-2504. 10.1101/gr.1239303

Singh M, Paul N, Singh S, and Nayak GR. 2018. Asthma and Fungus: Role in Allergic Bronchopulmonary Aspergillosis (ABPA) and Other Conditions. Indian J Pediatr 85:899-904. 10.1007/s12098-0182646-8

Thompson MD, Takasaki J, Capra V, Rovati GE, Siminovitch KA, Burnham WM, Hudson TJ, Bosse Y, and Cole DE. 2006. G-protein-coupled receptors and asthma endophenotypes: the cysteinyl leukotriene system in perspective. Mol Diagn Ther 10:353-366. 10.1007/BF03256212

UniProt Consortium T. 2018. UniProt: the universal protein knowledgebase. Nucleic Acids Res 46:2699. 10.1093/nar/gky092

PeerJ reviewing PDF | (2020:12:56161:2:0:NEW 19 Apr 2021) 
428 Van der Auwera GA, Carneiro MO, Hartl C, Poplin R, Del Angel G, Levy-Moonshine A, Jordan T, Shakir

429

430

431

432

433

434

435

436

437

438

439

440

441

442

443

444

445

446

447

448

449

450

451

452

453

454

455 Figure and Table legends

456

457

458

459

460 8:3409-3418. 10.1186/s12920-020-00785-y

K, Roazen D, Thibault J, Banks E, Garimella KV, Altshuler D, Gabriel S, and DePristo MA. 2013. From FastQ data to high confidence variant calls: the Genome Analysis Toolkit best practices pipeline. Curr Protoc Bioinformatics 43:11 10 11-11 10 33. 10.1002/0471250953.bi1110s43

Wang K, Li M, and Hakonarson H. 2010. ANNOVAR: functional annotation of genetic variants from highthroughput sequencing data. Nucleic Acids Res 38:e164. 10.1093/nar/gkq603

Zeller T, Wild P, Szymczak S, Rotival M, Schillert A, Castagne R, Maouche S, Germain M, Lackner K, Rossmann H, Eleftheriadis M, Sinning CR, Schnabel RB, Lubos E, Mennerich D, Rust W, Perret C, Proust C, Nicaud V, Loscalzo J, Hubner N, Tregouet D, Munzel T, Ziegler A, Tiret L, Blankenberg S, and Cambien F. 2010. Genetics and beyond--the transcriptome of human monocytes and disease susceptibility. PLoS One 5:e10693. 10.1371/journal.pone.0010693

Zerbino DR, Achuthan P, Akanni W, Amode MR, Barrell D, Bhai J, Billis K, Cummins C, Gall A, Giron CG, Gil L, Gordon L, Haggerty L, Haskell E, Hourlier T, Izuogu OG, Janacek SH, Juettemann T, To JK, Laird MR, Lavidas I, Liu Z, Loveland JE, Maurel T, McLaren W, Moore B, Mudge J, Murphy DN, Newman V, Nuhn M, Ogeh D, Ong CK, Parker A, Patricio M, Riat HS, Schuilenburg H, Sheppard D, Sparrow H, Taylor K, Thormann A, Vullo A, Walts B, Zadissa A, Frankish A, Hunt SE, Kostadima M, Langridge N, Martin FJ, Muffato M, Perry E, Ruffier M, Staines DM, Trevanion SJ, Aken BL, Cunningham F, Yates A, and Flicek P. 2018. Ensembl 2018. Nucleic Acids Res 46:D754-D761. 10.1093/nar/gkx1098

Zhang XY, Zhang LX, Tian CJ, Tang XY, Zhao LM, Guo YL, Cheng DJ, Chen XL, Ma LJ, and Chen ZC. 2016. LncRNAs BCYRN1 promoted the proliferation and migration of rat airway smooth muscle cells in asthma via upregulating the expression of transient receptor potential 1. Am J Transl Res

Zheng P, Huang C, Leng D, Sun B, and Zhang XD. 2020. Transcriptome analysis of peripheral whole blood identifies crucial lncRNAs implicated in childhood asthma. BMC Med Genomics 13:136.

Figure 1. Bioinformatic analysis pipeline for the transcriptome analysis of RNA-seq data.

Figure 2. Transcriptome-level mutational landscape of ABPA and asthma compared to health controls. A) Each row in the figure corresponds to one gene/lncRNA, whereas each column corresponds to one sample $(n=28)$. (blue bar represents ABPA patients, dark red bar represents

PeerJ reviewing PDF | (2020:12:56161:2:0:NEW 19 Apr 2021) 
461 asthma patients and light green represents health controls). (Top) Bar plots describing the 462 percentage of different type of alternations identified in each sample across all the identified 463 genes/lncRNAs. (Right) Bar plots of the percentage of different type of alternations of each 464 gene/lncRNAs across all the sample. (Bottom) bar plot represents the type of samples, blue bar 465 represents ABPA patients, dark red bar represents asthma patients and light green represents health 466 controls. B) The statistical analysis pipeline to detect whether the identified SSR event significantly existed between ABPA patients and health controls, as well as asthma patients and

468

469

470

471

472

473

474

475

476

477

478

479

480

481

482

483

484

485

486

487

488

489

490

491

492

493

494

495

health controls. C) The density distribution of the SSRs among the three types of samples. The graphs (D I) plot the SSRs difference between ABPA patients, asthma patients as well as health controls.

Figure 3. Differential expression analysis of ABPA, asthma patients and health controls. (A) Heatmap of all the differentially expressed transcripts identified by the comparison of ABPA with health controls and asthma with health controls. (B) Functional enrichment analysis based on the differentially expressed transcripts identified by the comparison of asthma with health controls. (C) Functional enrichment analysis based on the differentially expressed transcripts identified by the comparison of ABPA with health controls. (D) Overlap of the differentially expressed transcripts between two comparisons. (E) Functional enrichment analysis of the differentially expressed transcripts for both diseases.

Figure 4. Cluster analysis of differentially expressed mRNAs and IncRNAs in all human blood samples. (A) Cluster 1 indicates the mRNAs and lncRNAs that were down-regulation expressed in health controls but were up-regulation in ABPA and asthma patients. (B) Bar plot shows the Gene ontology (GO) functional enrichment analysis based on the mRNAs of cluster1. (C) Cluster 2 indicates the mRNAs and lncRNAs that specifically up-regulation expressed in ABPA patients. (D) Bar plot shows the Gene ontology (GO) functional enrichment analysis based on the mRNAs of cluster2. (E) Cluster 3 indicates the mRNAs and lncRNAs that were upregulation expressed in health controls but were down-regulation in ABPA and asthma patients. (F) Bar plot shows the Gene ontology (GO) functional enrichment analysis based on the mRNAs of cluster3.

Figure 5. Differential network analysis between ABPA, asthma patients and health controls. (A) Visualization of the immune-related loss-of-function network of ABPA patients $\left(\left|\mathrm{PCC}_{\text {healthy }}\right| \geq\right.$ $0.95,\left|\mathrm{PCC}_{\mathrm{ABPA}}\right| \leq 0.30,\left|\mathrm{PCC}_{\mathrm{ABPA} \text { - healthy }}\right| \geq 1.00$ ). (B) Visualization of the immune-related loss-offunction network of asthma patients $\left(\left|\mathrm{PCC}_{\text {healthy }}\right| \geq 0.93,\left|\mathrm{PCC}_{\mathrm{AS}}\right| \leq 0.30,\left|\mathrm{PCC}_{\mathrm{AS}-\text { healthy }}\right| \geq 1.00\right)$. 
496 (C) Visualization of the immune-related gain-of-function network of ABPA patients $\left(\left|\mathrm{PCC}_{\text {healthy }}\right|\right.$ $\left.497 \leq 0.30,\left|\mathrm{PCC}_{\mathrm{ABPA}}\right| \geq 0.80,\left|\mathrm{PCC}_{\mathrm{ABPA} \text { - healthy }}\right| \geq 1.00\right)$. (D) Visualization of the immune-related gain498 of-function network of asthma patients $\left(\left|\mathrm{PCC}_{\text {healthy }}\right| \leq 0.30,\left|\mathrm{PCC}_{\mathrm{AS}}\right| \geq 0.90,\left|\mathrm{PCC}_{\mathrm{AS} \text { - healthy }}\right| \geq 1.00\right)$. 499 (E) Visualization of the immune-related anti-function network of ABPA patients $\left(\left|\mathrm{PCC}_{\text {healthy }}\right| \geq\right.$ $5000.70,\left|\mathrm{PCC}_{\mathrm{ABPA}}\right| \geq 0.70, \mid \mathrm{PCC}_{\mathrm{ABPA}}$ - healthy $\mid \geq 1.00$ ). (F) Visualization of the immune-related anti501 function network of asthma patients $\left(\left(\left|\mathrm{PCC}_{\text {healthy }}\right| \geq 0.70,\left|\mathrm{PCC}_{\mathrm{AS}}\right| \geq 0.70,\left|\mathrm{PCC}_{\mathrm{AS} \text { - healthy }}\right| \geq 1.00\right)\right)$. 502

Figure 6. Functional analysis and GEO validation of key IncRNAs (hub nodes) derived from the loss-of-function networks of two diseases. (A) hub node: SREBF1-208. (B) hub node: PUF60-218. (C) hub node: HNRNPUL1-210.

(D) hub node: AL139423.1-201. (E) hub node: AC106028.4-201. (Left) sub-networks display all the target mRNAs for the key lncRNAs. (Right) bar plots show Gene ontology (GO) functional enrichment analysis based on the corresponding target mRNAs. F-I. Validation of the expression level of four selected hub nodes (ENST00000327423, ENST00000369443, ENST00000587128 and ENST00000595806) between asthmatic groups and health controls. (F) Validation based on microarray data of GSE35571. (G)

511 Validation based on microarray data of GSE473. (H) Validation based on microarray data of 512 GSE31773. (I) Validation based on microarray data of GSE2125.

513

514 Table 1. Baseline characteristics of the study population.

516 Table 2. Basic statistics of variants occurred in RNA-seq data detected by GATK analysis 517 pipeline.

518

519 Table 3. Hub nodes of the common immune-related functional network in ABPA and 520 asthma. 
Figure 1

\section{Bioinformatic analysis pipeline for the transcriptome analysis of RNA-seq data}

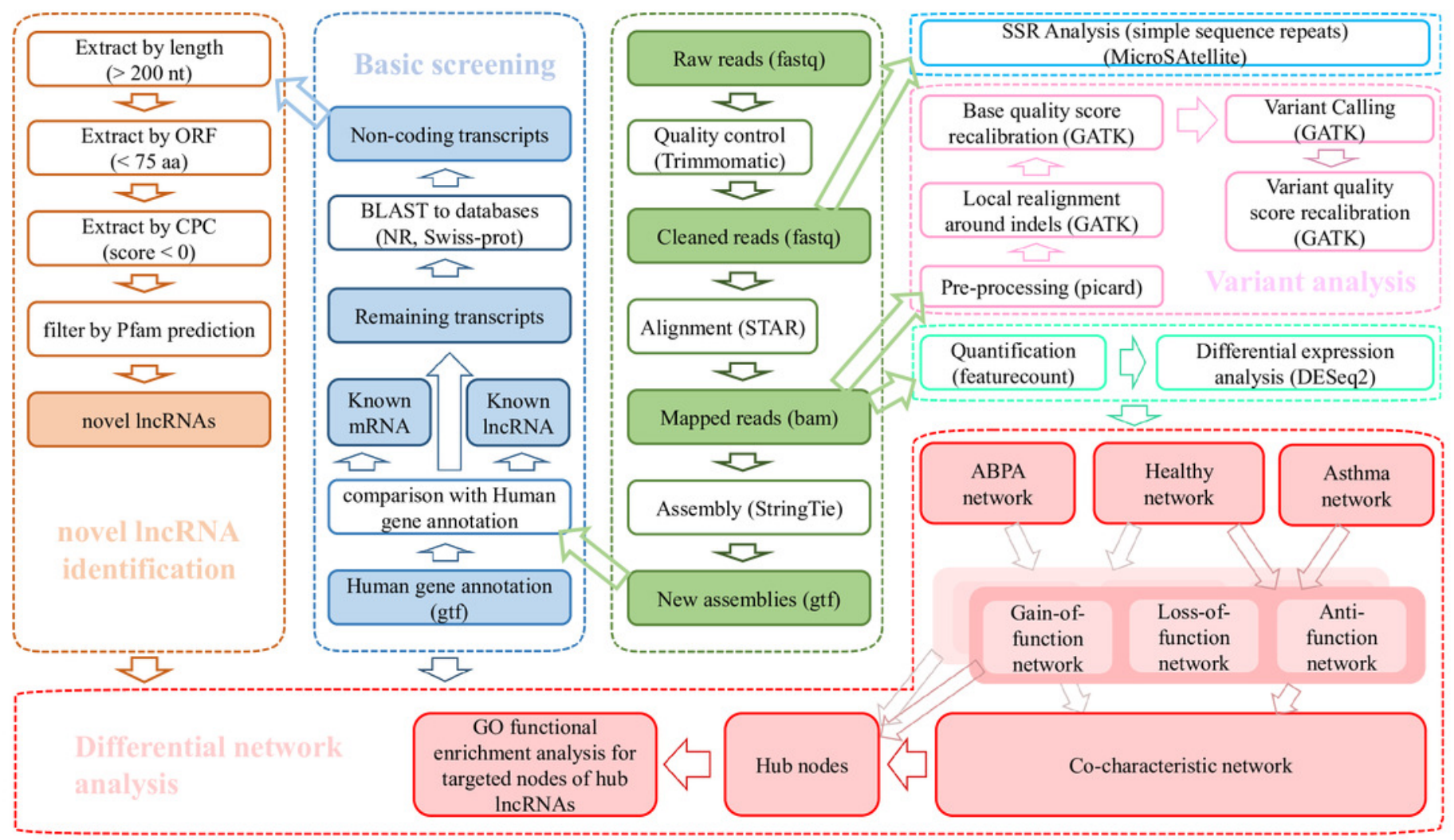




\section{Figure 2}

Transcriptome-level mutational landscape of ABPA and asthma compared to health controls.

A) Each row in the figure corresponds to one gene/InCRNA, whereas each column corresponds to one sample $(n=28)$. (blue bar represents ABPA patients, dark red bar represents asthma patients and light green represents health controls). (Top) Bar plots describing the percentage of different type ofalternations identified in each sample across all the identified genes/IncRNAs. (Right) Bar plots of the percentage of different type of alternations of each gene/IncRNAs across all the sample. (Bottom) bar plot represents the type of samples, blue bar represents ABPA patients, dark red bar represents asthma patients and light green represents health controls. B) The statistical analysis pipeline to detect whether the identified SSR event significantly existed between ABPA patients and health controls, as well as asthma patients and health controls. C) The density distribution of the SSRs among the three types of samples. The graphs (D I) plot the SSRs difference between ABPA patients, asthma patients as well as health controls. 
A

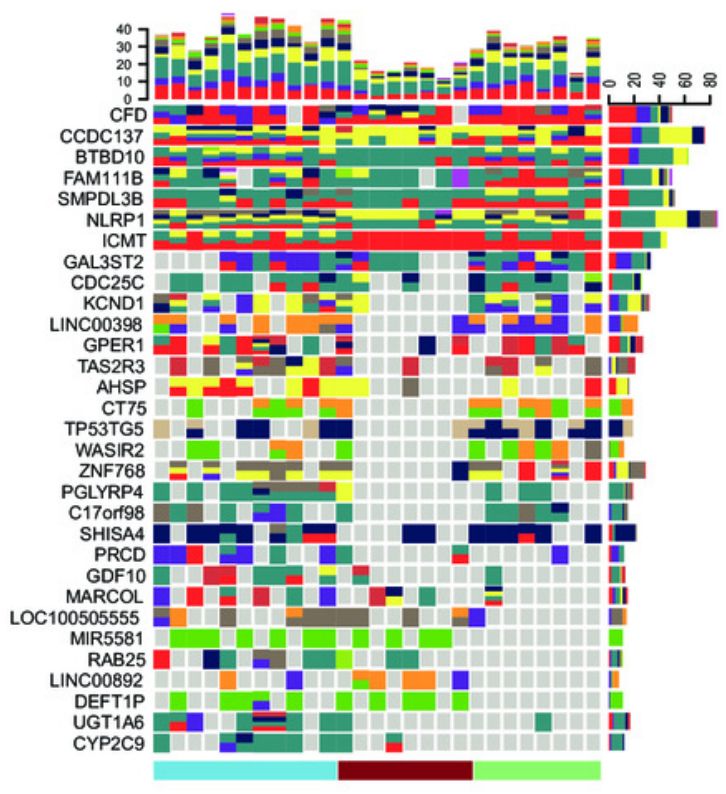

D

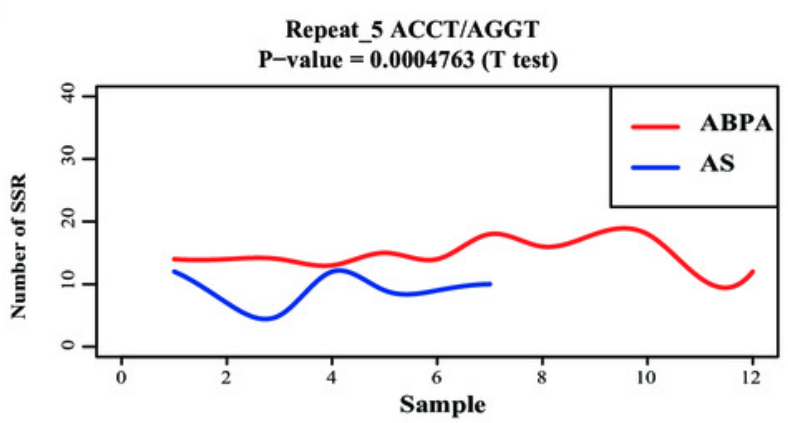

F

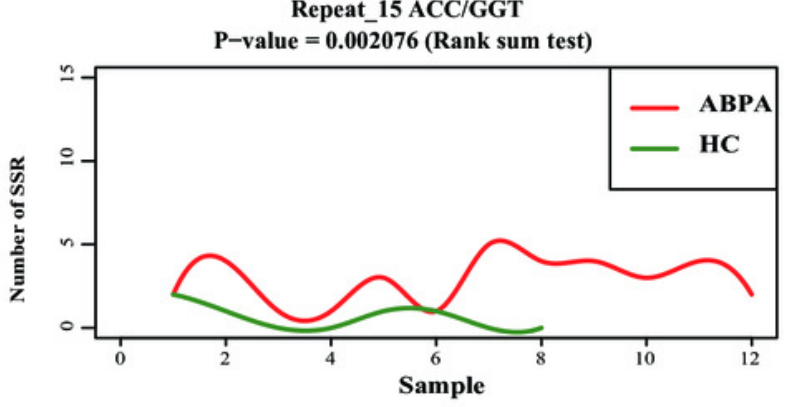

H

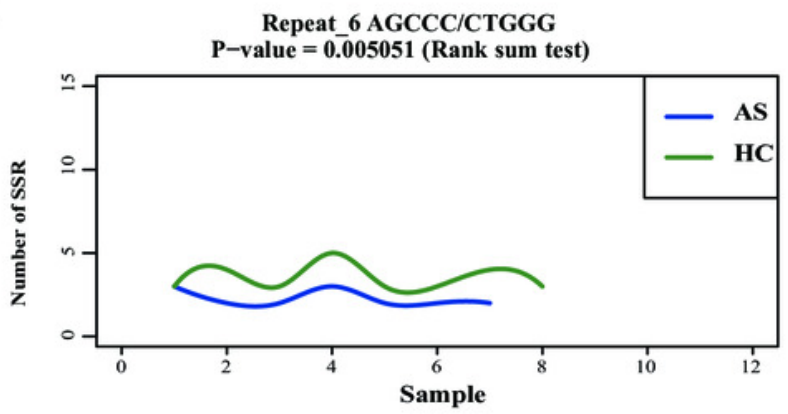

B

\section{Alternations}

UTR3

ncRNA_exonic

Irameshift insertion
synonymous SNV

ncRNA_intronic

stopgain

nonframeshift deletion

frameshift deletion

- downstream

nonsynonymous SNV

intronic

ABPA

Health Control

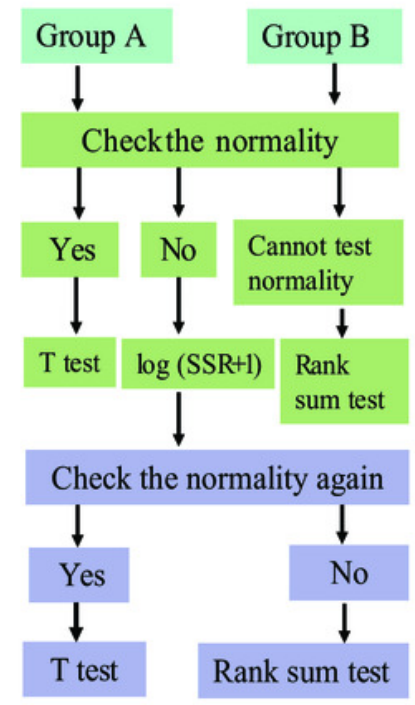

C

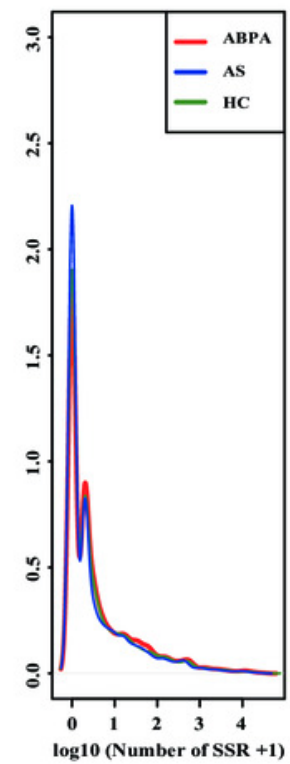

$\mathbf{E}$

Repeat_24 AG/CT $\mathrm{P}$-value $=0.0005386$ ( $\mathrm{T}$ test $)$

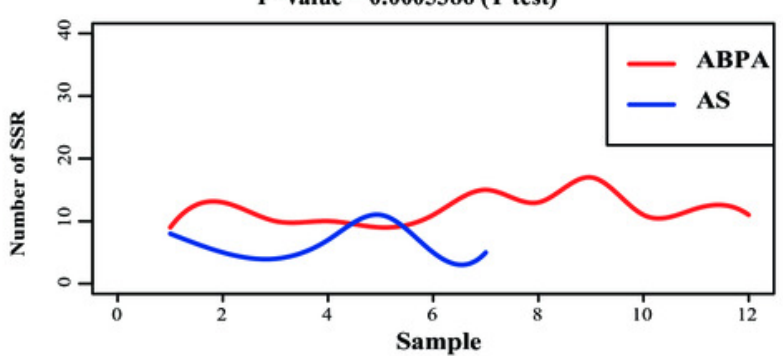

G
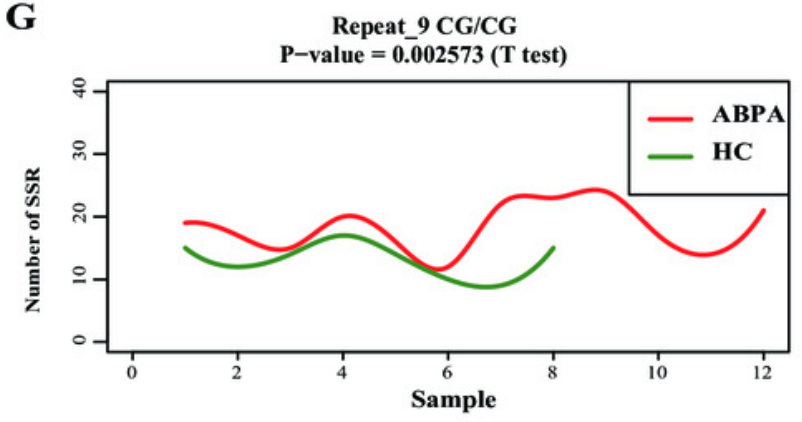

I

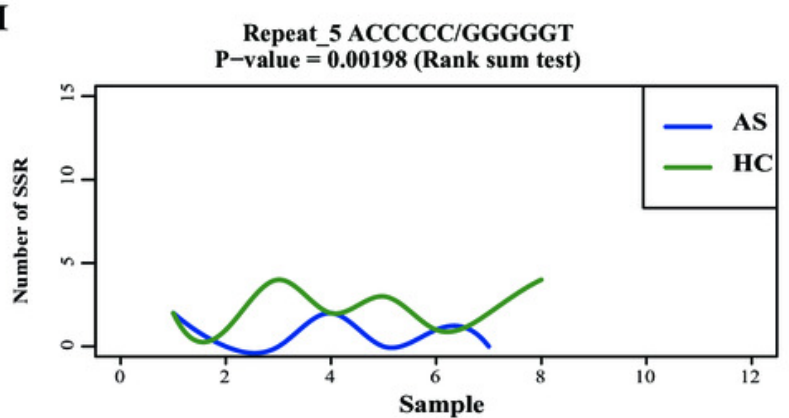




\section{Figure 3}

Differential expression analysis of ABPA, asthma patients and health controls.

(A) Heatmap of all the differentially expressed transcripts identified by the comparison of ABPA with health controls and asthma with health controls. (B) Functional enrichment analysis based on the differentially expressed transcripts identified by the comparison of asthma with health controls. (C) Functional enrichment analysis based on the differentially expressed transcripts identified by the comparison of ABPA with health controls. (D) Overlap of the differentially expressed transcripts between two comparisons. (E) Functional enrichment analysis of the differentially expressed transcripts for both diseases. 
A

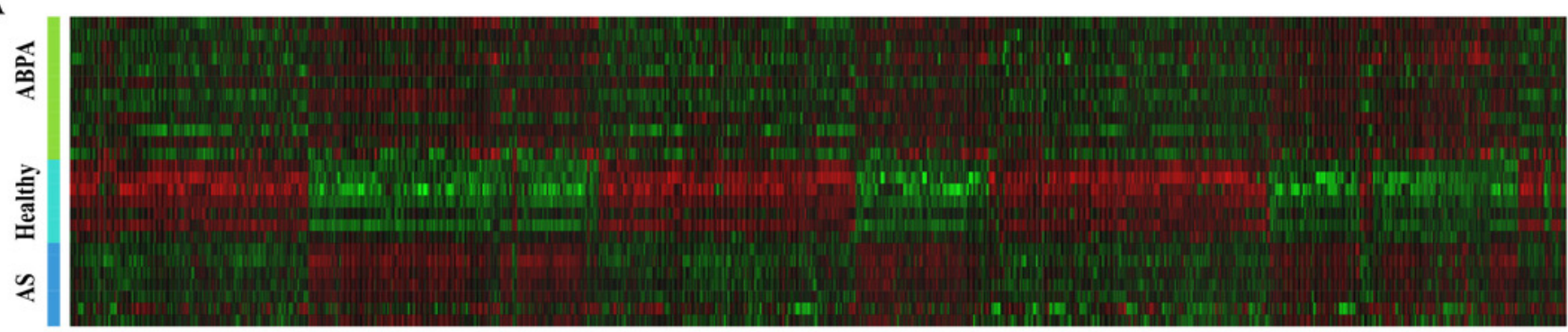

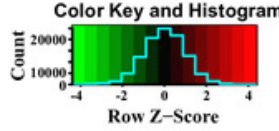

B

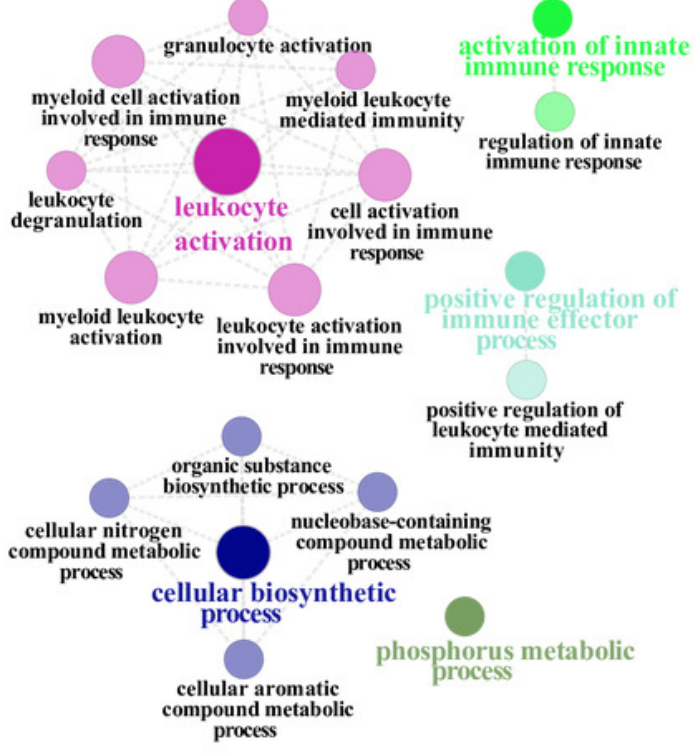

D

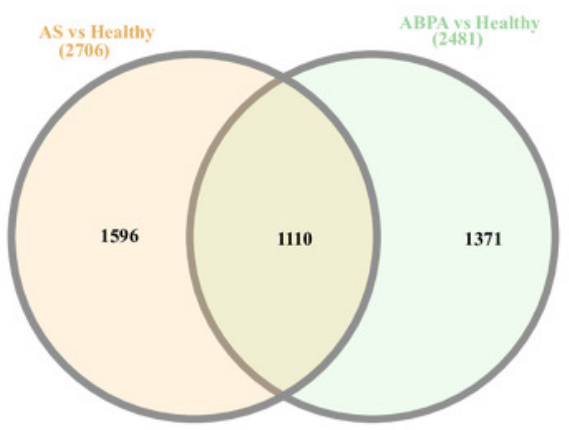

C

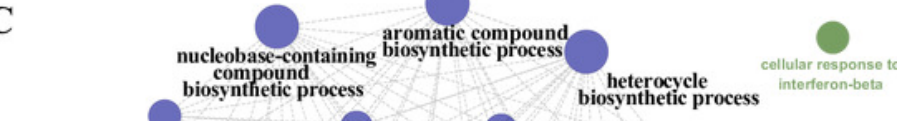
regulation of cellular $\begin{gathered}\text { organic cyclic } \\ \text { biosynthetic process } \\ \text { compound } \\ \text { biosynthetic process }\end{gathered}$
$\begin{gathered}\text { cellular } \\ \text { macromolecule }\end{gathered}$ cellular nitrogen
compound
biosynthetic process
compound metabolic $\quad \begin{aligned} & \text { regulation of RNA } \\ & \text { metabolic process }\end{aligned}$

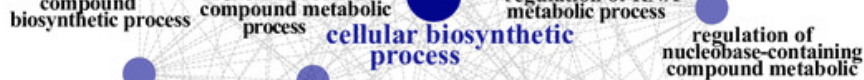
$\begin{gathered}\text { nucleic acid-templated } \\ \text { transcription } \\ \text { transcription by } \\ \text { polymerase }\end{gathered}$
nucleobase-containing transcription by RNA $\begin{gathered}\text { nucleobase-containing } \\ \text { compound metabolic } \\ \text { process }\end{gathered}$
$\begin{gathered}\text { transcription by RNA } \\ \text { polymerase II }\end{gathered}$ $\begin{gathered}\begin{array}{c}\text { process } \\ \text { transcription, } \\ \text { DNA-templated }\end{array} \\ \end{gathered}$ heterocycle metabolic
process organic cyclic organic cyclic
compound metabolic

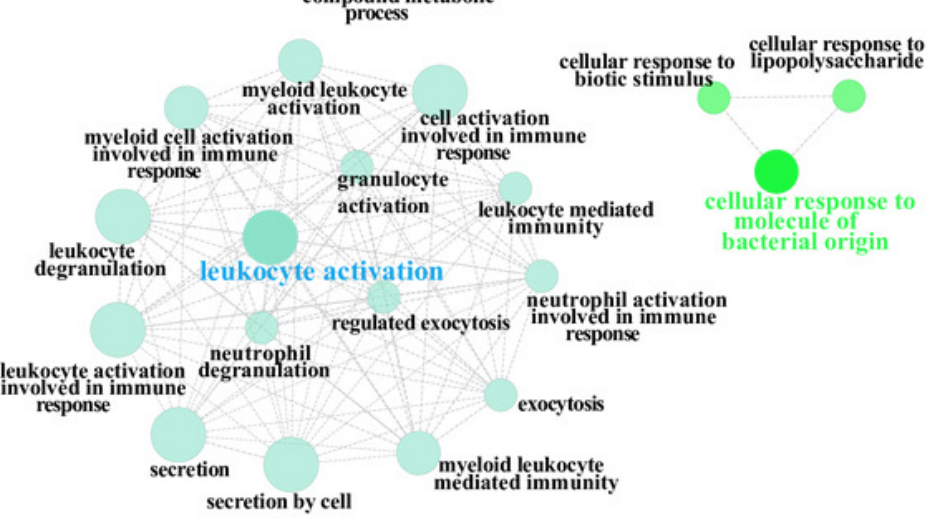

$\mathbf{E}$

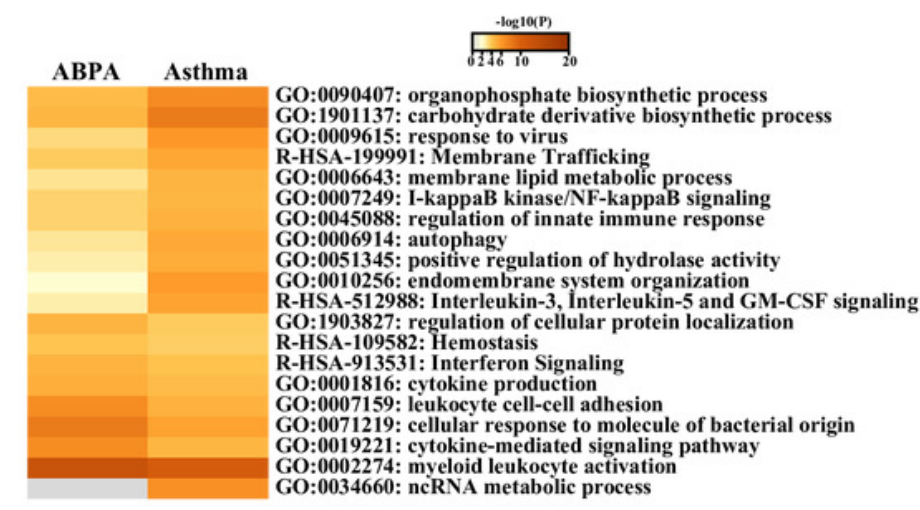




\section{Figure 4}

Cluster analysis of differentially expressed mRNAs and IncRNAs in all human blood samples

(A) Cluster 1 indicates the mRNAs and IncRNAs that were down-regulation expressed in health controls but were up-regulation in ABPA and asthma patients. (B) Bar plot shows the Gene ontology (GO) functional enrichment analysis based on the mRNAs of cluster1. (C) Cluster 2 indicates the mRNAs and IncRNAs that specifically up-regulation expressed in ABPA patients. (D) Bar plot shows the Gene ontology (GO) functional enrichment analysis based on the mRNAs of cluster2. (E) Cluster 3 indicates the mRNAs and IncRNAs that were upregulation expressed in health controls but were down-regulation in ABPA and asthma patients. (F) Bar plot shows the Gene ontology (GO) functional enrichment analysis based on the mRNAs of cluster3. 
A

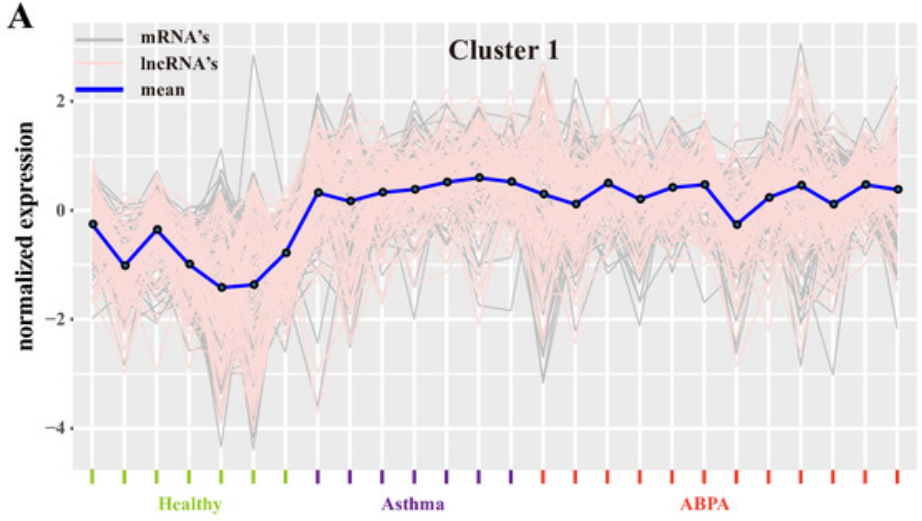

C

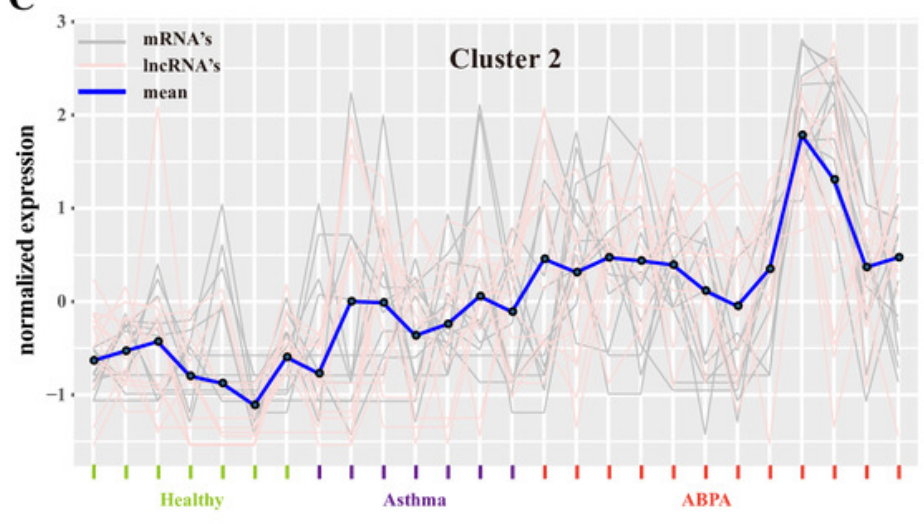

E

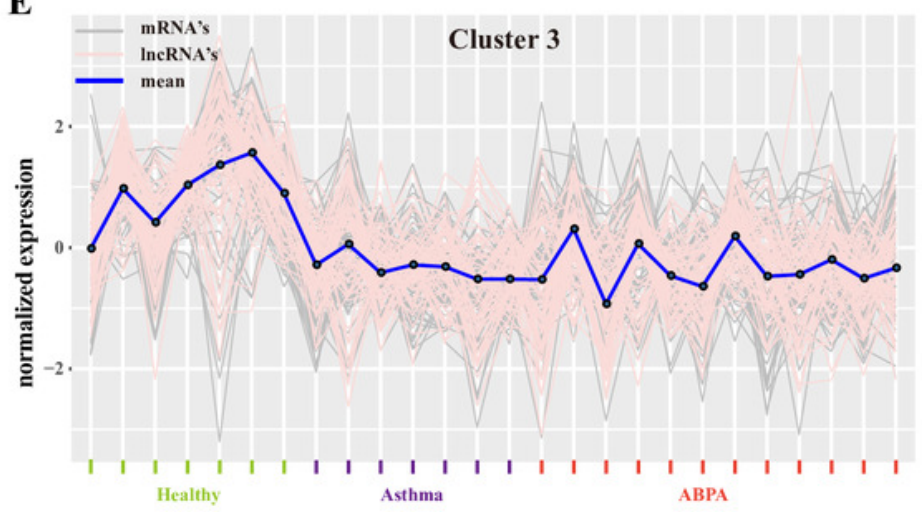

B GO Biological Processes KEGG Pathway Reactome Gene Sets Antiviral mechanism by IFN-stimulated genes -

myeloid leukocyte mediated immunity -

Nucleotide excision repair -

peptidyl-lysine trimethylation -

nucleic acid phosphodiester bond hydrolysis -

regulation of defense response to virus negative regulation of myeloid -
leukocyte differentiation

DAP12 interactions -

Nucleobase biosynthesis -

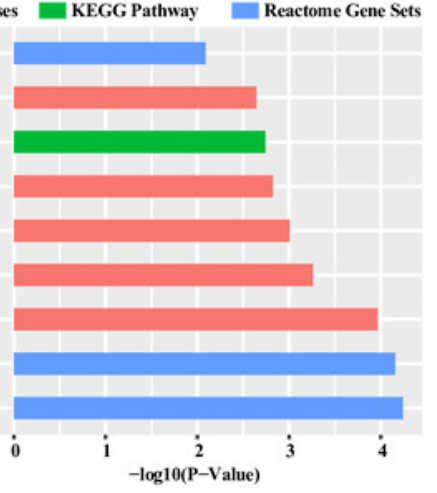

D GO Biological Processes

supramolecular fiber organization positive regulation of organelle organization -

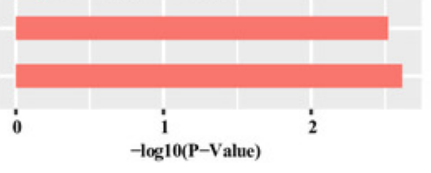

\section{F}

columnar/cuboidal epithelial cell differentiation -

blood vessel development -

negative regulation of cell-cell adhesion -

Cytokine-cytokine receptor interaction -

positive regulation of neurogenesis -

regulation of innate immune response -

Sphingolipid metabolism -

positive regulation of immune effector process -

regulation of apoptotic signaling pathway -

peptidyl-tyrosine phosphorylation -

NIK/NF-kappaB signaling -

myeloid dendritic cell differentiation -

regulation of canonical Wnt signaling pathway -

cytokine-mediated signaling pathway -

cytokine production -

immune response-regulating cell surface

receptor signaling pathway -
recent sulace

lymphocyte activation involved -
in immune response

leukes immune response

leukocyte activation involved -
in immune response

T cell activation -

KEGG Pathway Reactome Gene Sets
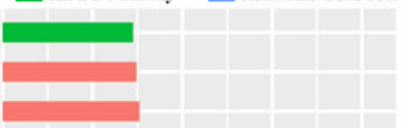


\section{Figure 5}

Differential network analysis between ABPA, asthma patients and health controls

(A) Visualization of the immune-related loss-of-function network of ABPA patients ( $\left|P C C_{\text {healthy }}\right|$ $\geq 0.95,\left|P C C_{A B P A}\right| \leq 0.30, \mid P C C_{A B P A}$ - healthy $\mid \geq 1.00$ ). (B) Visualization of the immune-related lossof-function network of asthma patients $\left(\left|P C C_{\text {nealthy }}\right| \geq 0.93,\left|P C C_{A S}\right| \leq 0.30, \mid P C C_{A S}\right.$ - heathy $\mid \geq$ 1.00). (C) Visualization of the immune-related gain-of-function network of ABPA patients $\left(\left|P C C_{\text {heatthy }}\right| \leq 0.30 \square\left|P C C_{A B P A}\right| \geq 0.80, \mid P C C_{A B P A}\right.$ - heathy $\left.\mid \geq 1.00\right)$. (D) Visualization of the immunerelated gain-of-function network of asthma patients ( $\left|P C C_{\text {heatthy }}\right| \leq 0.30,\left|P C C_{A S}\right| \geq 0.90, \mid P C C_{A S}$. heatthy $\geq 1.00$ ). (E) Visualization of the immune-related anti-function network of ABPA patients $\left(\left|P C C_{\text {heatthy }}\right| \geq 0.70,\left|P C C_{A B P A}\right| \geq 0.70,\left|P C C_{A B P A \text { - heathy }}\right| \geq 1.00\right)$. (F) Visualization of the immunerelated anti-function network of asthma patients ( $\left|P C C_{\text {nealthy }}\right| \geq 0.70,\left|P C C_{A S}\right| \geq 0.70, \mid P C C_{A s}$. healthy $\mid \geq 1.00)$ ). 


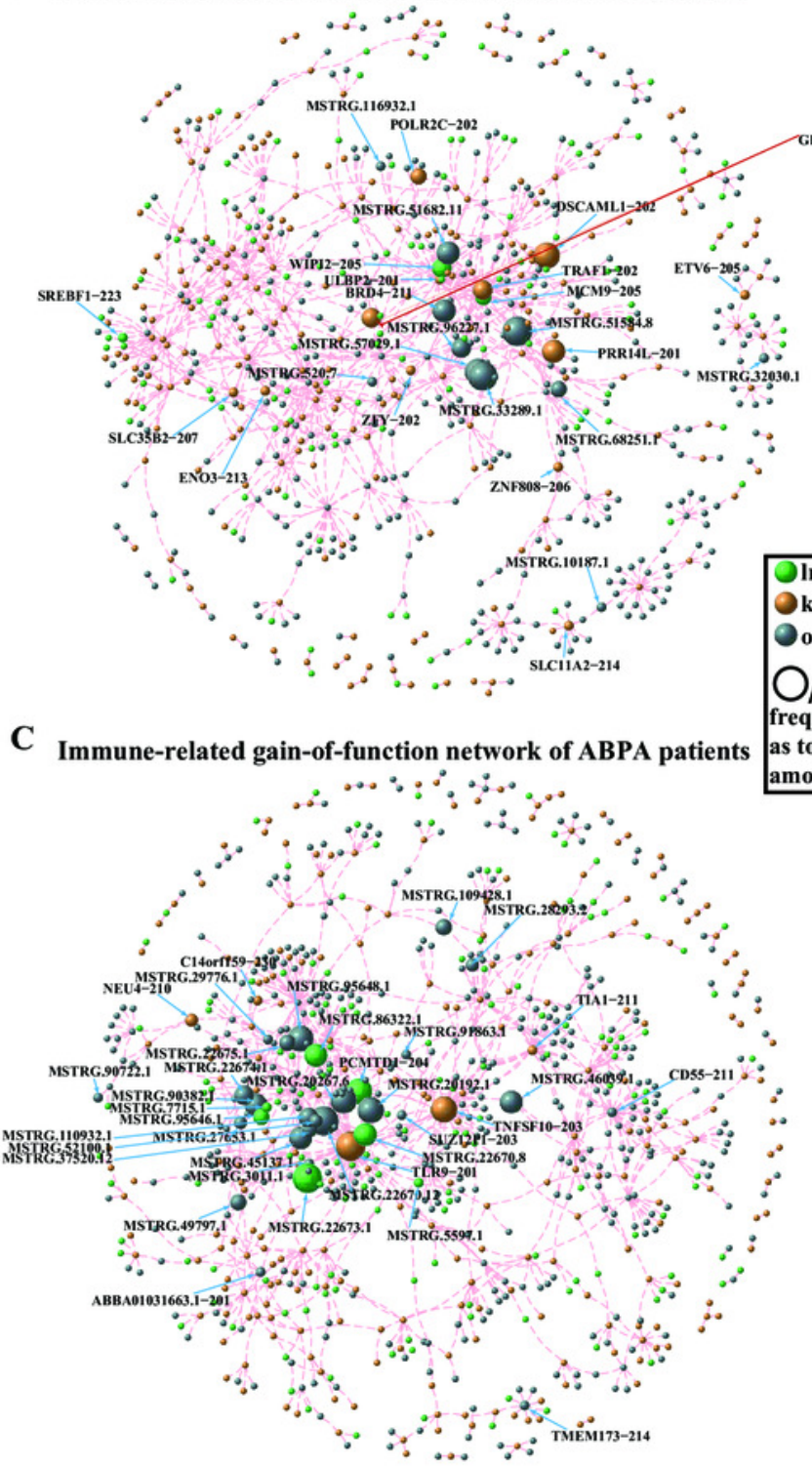

E Immune-related anti-function network of ABPA patients $\because \because \because \because \because \because \because \because \cdots \cdots \cdots \cdots$

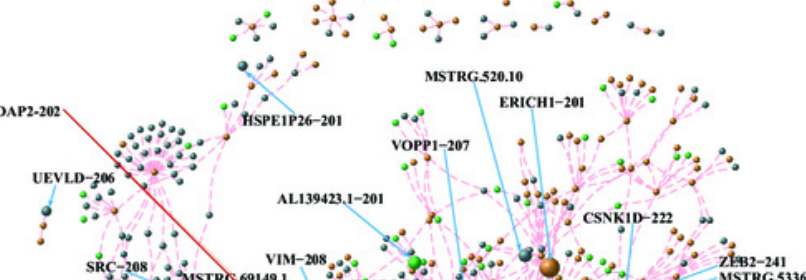

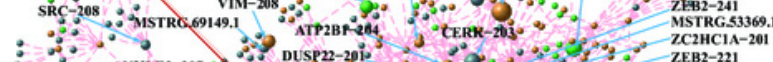

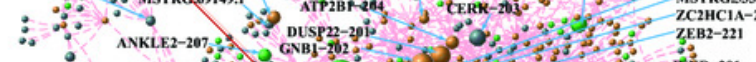

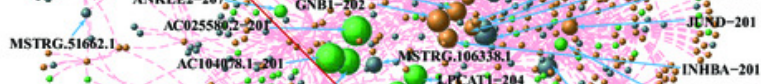

CCRL2-202, $\cdots$

CCRL2-202,

IncRNA
known mRNA
others

$\mathrm{O} / \mathrm{O} / \mathrm{o}$

(requency of counting

as top 20 hub nodes

ng 12 algorithms

D $_{\text {Im }}$

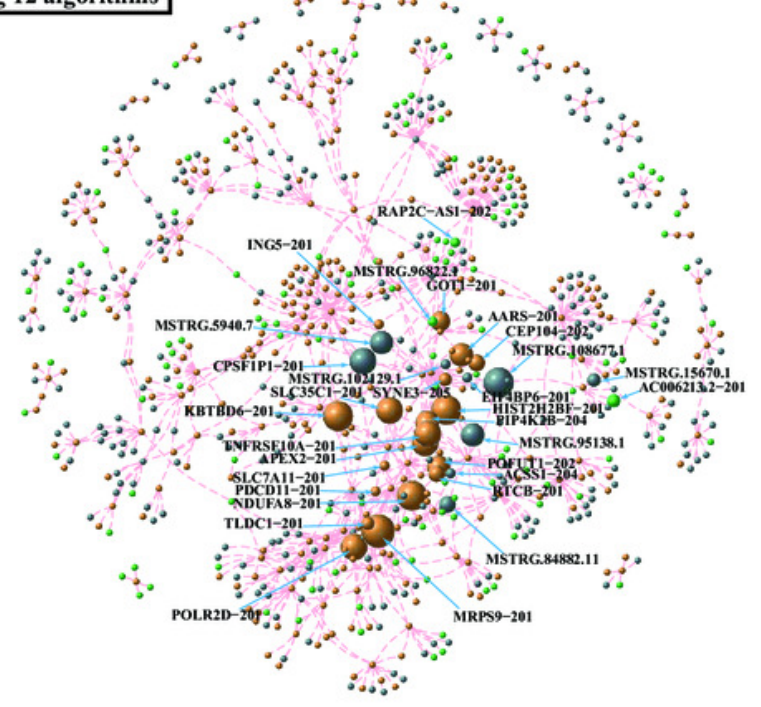

F Immune-related anti-function network of asthma patients

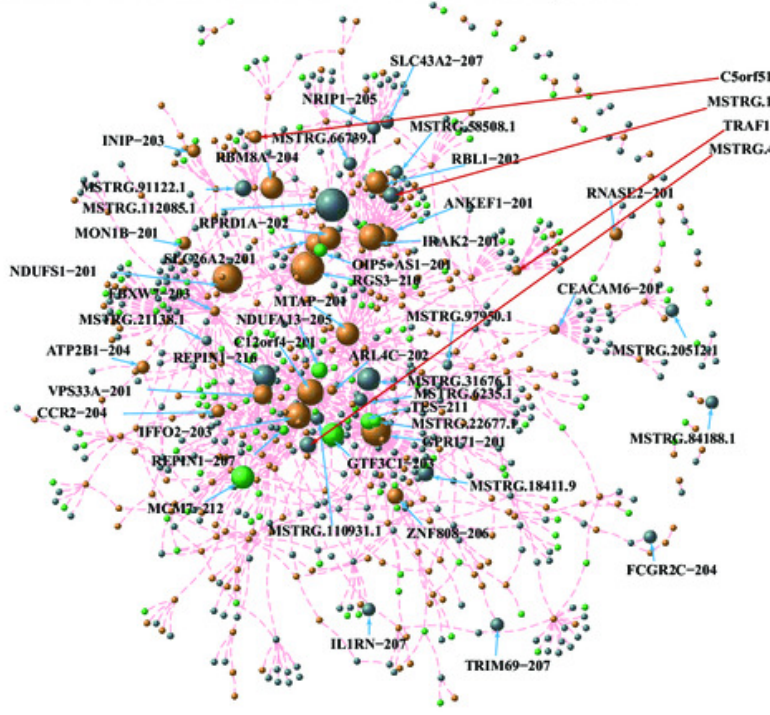




\section{Figure 6}

Functional analysis and GEO validation of key IncRNAs (hub nodes) derived from the loss-of-function networks of two diseases

(A) hub node: SREBF1-208. (B) hub node: PUF60-218. (C) hub node: HNRNPUL1-210. (D) hub node: AL139423.1-201. (E) hub node: AC106028.4-201. (Left) sub-networks display all the target mRNAs for the key IncRNAs. (Right) bar plots show Gene ontology $(G O)$ functional enrichment analysis based on the corresponding target mRNAs. F-I. Validation of the expression level of four selected hub nodes (ENST00000327423, ENST00000369443, ENST00000587128 and ENST00000595806) between asthmatic groups and health controls. (F) Validation based on microarray data of GSE35571. (G) Validation based on microarray data of GSE473. (H) Validation based on microarray data of GSE31773. (I) Validation based on microarray data of GSE2125. 

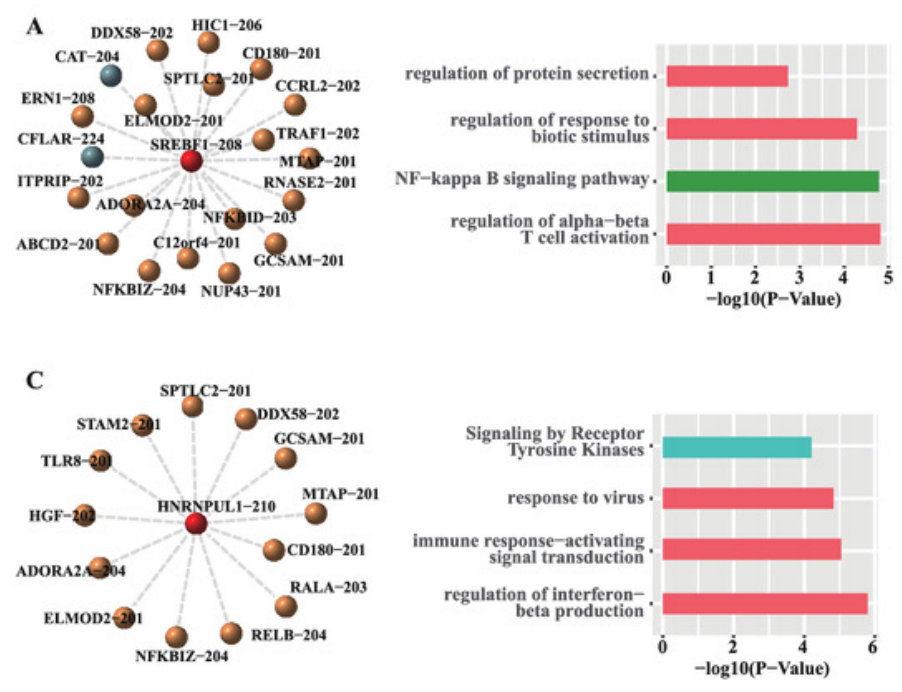

E

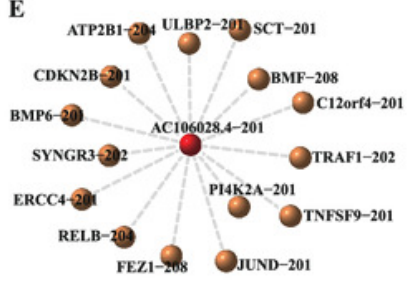

F

ENST00000327423

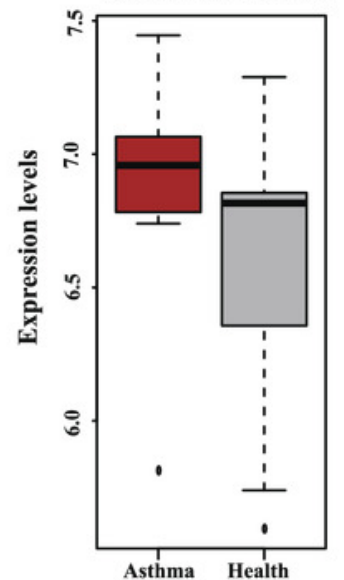

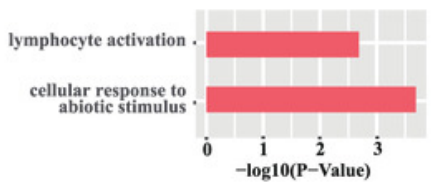

G

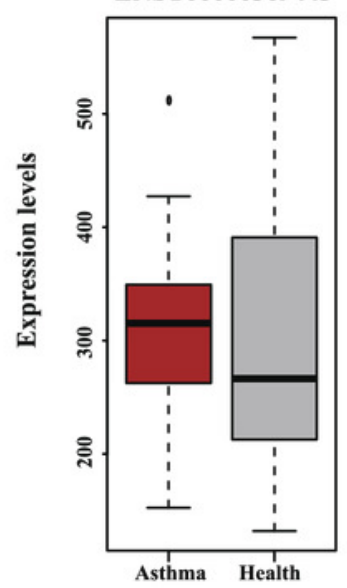

B
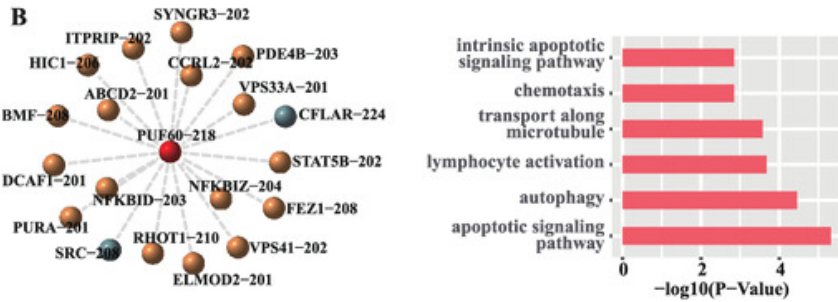

D

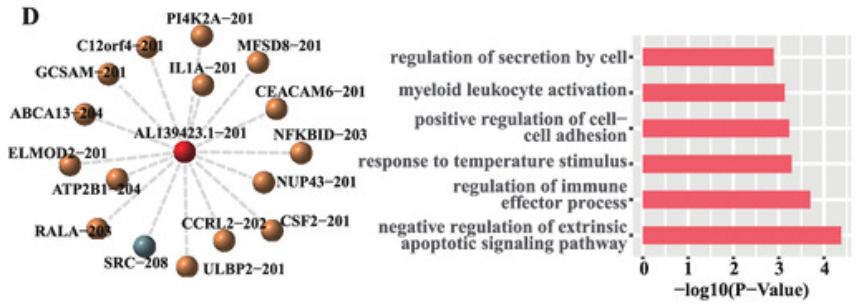

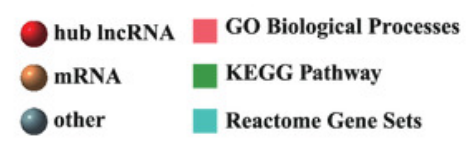

H

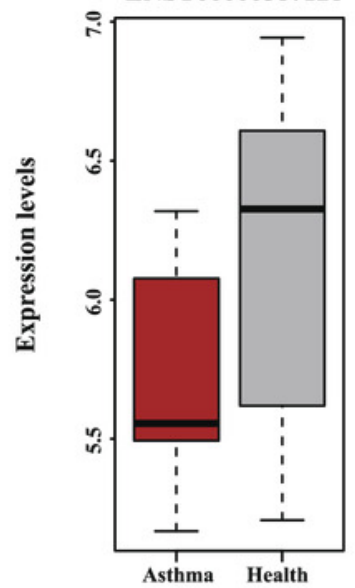


Table $\mathbf{1}$ (on next page)

Baseline characteristics of the study population 
1 Table 1. Baseline characteristics of the study population.

\begin{tabular}{|c|c|c|c|c|}
\hline & Asthma $(n=7)$ & $\operatorname{ABPA}(n=12)$ & $\begin{array}{l}\text { Healthy control } \\
\qquad(\mathrm{n}=8)\end{array}$ & $P$ value \\
\hline Age (years) & $44[41,46]$ & $34[29,40.75]^{*}$ & $26.50[23.25,35.75]^{*}$ & 0.007 \\
\hline \multicolumn{5}{|l|}{ Blood cell count } \\
\hline Eosinophil $\left(\times 10^{9}\right.$ cells $\left./ \mathrm{L}\right)$ & $0.36[0.05,0.98]$ & $0.58[0.33,0.90]$ & $0.20[0.10,0.35]$ & 0.284 \\
\hline Neutrophil $\left(\times 10^{9}\right.$ cells $\left./ \mathrm{L}\right)$ & $5.30[4.00,7.50]$ & $5.00[3.40,7.40]$ & $3.60[3.20,4.35]$ & 0.208 \\
\hline \multicolumn{5}{|l|}{ Induced sputum } \\
\hline Neutrophil (\%) & $79.00[47.00,88.50]$ & $39.75[14.25,76.88]$ & - & 0.297 \\
\hline Macrophage (\%) & $9.00[7.80,12.00]$ & $33.75[0.13,79.75]$ & - & 0.999 \\
\hline Eosinophil (\%) & $11.50[0.50,40.50]$ & $8.00[1.38,39.00]$ & - & 0.999 \\
\hline Lymphocytes (\%) & $1.00[0.50,1.80]$ & $1.50[0.50,5.13]$ & - & 0.655 \\
\hline \multicolumn{5}{|l|}{ Immunological characteristic } \\
\hline Total IgE level (kUA/L) & $529[40.2,826]$ & $2595[695.5,4757]^{*}$ & - & 0.040 \\
\hline sIgE positivity $(\%)$ & $57.1 \%$ & $100 \% *$ & - & 0.013 \\
\hline A.f $\operatorname{sIgE}$ positivity (\%) & $14.3 \%$ & $100 \% * *$ & - & $<0.001$ \\
\hline \multicolumn{5}{|l|}{ Glucocorticoids } \\
\hline Use of oral glucocorticoids (\%) & $42.9 \%$ & $58.3 \%$ & - & 0.515 \\
\hline $\begin{array}{l}\text { Use of inhaled glucocorticoids } \\
(\%)\end{array}$ & $71.4 \%$ & $50 \%$ & - & 0.361 \\
\hline
\end{tabular}

2 Data were given as medians with interquartile range (IQR). ${ }^{*} P<0.05$ compared with asthma.

$3 * * P<0.01$ compared with asthma.

4 


\section{Table 2 (on next page)}

Basic statistics of variants occurred in RNA-seq data detected by GATK analysis pipeline. 
Table 2. Basic statistics of variants occurred in RNA-seq data detected by GATK analysis pipeline.

\begin{tabular}{cc}
\hline Variant type & number \\
\hline UTR5; UTR3 & 55 \\
UTR5 & 17,091 \\
UTR3 & 147,976 \\
upstream;downstream & 2,484 \\
upstream & 39,078 \\
downstream & 63,520 \\
splicing & 2,523 \\
exonic & 62,680 \\
exonic; splicing & 53 \\
intergenic & $1,359,937$ \\
intronic & $3,170,032$ \\
ncRNA_exonic & 33,883 \\
ncRNA_exonic;splicin & 22 \\
g & \\
ncRNA_intronic & 293,956 \\
ncRNA_splicing & 177 \\
ncRNA_UTR5 & 1 \\
\hline
\end{tabular}




\section{Table 3 (on next page)}

Hub nodes of the common immune-related functional network in ABPA and asthma. 
Table 3. Hub nodes of the common immune-related functional network in ABPA and asthma.

\begin{tabular}{|c|c|c|c|c|c|c|}
\hline Transcript ID & Gene name & Type & $\begin{array}{l}\text { Differential } \\
\text { expression } \\
\text { of } \\
\text { regulation } \\
\text { in asthma }\end{array}$ & $\begin{array}{l}\text { Differential } \\
\text { expression } \\
\text { of } \\
\text { regulation } \\
\text { in ABPA }\end{array}$ & $\begin{array}{r}\text { frequency } \\
\text { of counting } \\
\text { as top } 20 \\
\text { hub nodes } \\
\text { among } 12 \\
\text { algorithms }\end{array}$ & $P$ value \\
\hline $\begin{array}{l}\text { ENST0000032742 } \\
3\end{array}$ & PRR14L-201 & mRNA & UP & UP & 8 & 0 \\
\hline MSTRG.89849.1 & MSTRG.89849.1 & other & DOWN & DOWN & 8 & 0 \\
\hline $\begin{array}{l}\text { ENST0000060680 } \\
2\end{array}$ & AL139423.1-201 & $\operatorname{lncRNA}$ & DOWN & DOWN & 8 & 0 \\
\hline $\begin{array}{l}\text { ENST0000047747 } \\
5\end{array}$ & SRC-208 & other & UP & DOWN & 8 & 0.98 \\
\hline $\begin{array}{l}\text { ENST0000039903 } \\
6\end{array}$ & CCRL2-202 & mRNA & UP & DOWN & 8 & 0.98 \\
\hline MSTRG.38792.1 & MSTRG.38792.1 & other & UP & DOWN & 8 & 0 \\
\hline $\begin{array}{l}\text { ENST0000036944 } \\
3\end{array}$ & GDAP2-202 & mRNA & UP & UP & 8 & 0 \\
\hline MSTRG.68250.13 & MSTRG.68250.1 & other & UP & DOWN & 8 & 0 \\
\hline MSTRG.33289.1 & MSTRG.33289.1 & other & DOWN & DOWN & 8 & 0 \\
\hline $\begin{array}{l}\text { ENST0000052064 } \\
3\end{array}$ & SPAG1-206 & mRNA & DOWN & DOWN & 7 & 0 \\
\hline MSTRG.520.10 & MSTRG.520.10 & other & DOWN & DOWN & 7 & 0 \\
\hline MSTRG.69149.13 & MSTRG.69149.1 & other & UP & DOWN & 6 & 0 \\
\hline $\begin{array}{l}\text { ENST0000059580 } \\
6\end{array}$ & HNRNPUL1-210 & $\operatorname{lncRNA}$ & DOWN & DOWN & 6 & 0 \\
\hline $\begin{array}{l}\text { ENST0000061450 } \\
9\end{array}$ & AC106028.4-201 & $\operatorname{lncRNA}$ & DOWN & DOWN & 6 & 0 \\
\hline $\begin{array}{l}\text { ENST0000061963 } \\
6\end{array}$ & SLC35B2-207 & mRNA & UP & DOWN & 6 & 0 \\
\hline $\begin{array}{l}\text { ENST0000053199 } \\
5\end{array}$ & PUF60-218 & lncRNA & UP & DOWN & 5 & 0 \\
\hline $\begin{array}{l}\text { ENST0000051438 } \\
5\end{array}$ & FXYD2-204 & other & UP & DOWN & 5 & 0 \\
\hline MSTRG.61564.1 & MSTRG.61564.1 & other & UP & DOWN & 5 & 0 \\
\hline
\end{tabular}




\begin{tabular}{lllllll} 
ENST0000049073 & UEVLD-206 & other & UP & DOWN & 4 & 0 \\
6 & & & & & 4 & 0 \\
MSTRG.111947.1 & MSTRG.111947.1 & other & UP & DOWN & 4 & 0 \\
ENST0000046935 & SREBF1-208 & lncRNA & DOWN & DOWN & 4 \\
6 & & & UP & DOWN & 3 & 0 \\
MSTRG.96227.1 & MSTRG.96227.1 & other & UP & DOWN & 3 & 0 \\
MSTRG.68251.13 & MSTRG.68251.1 & other & UP & DOWN \\
\hline
\end{tabular}

${ }^{3}$ : the transcript exists in the cluster 3 of cluster analysis in Figure 5E. 